\title{
An Efficient Cooperative Media Access Control Based Relay Node Selection In Wireless Networks
}

\author{
Christo Ananth ${ }^{1}$, Dr. G. Arul Dalton ${ }^{2}$, Dr.S.Selvakani ${ }^{3}$ \\ Assistant Professor, Department of Electronics \& Communication Engineering, \\ Francis Xavier Engineering College, Tirunelveli, India ${ }^{1}$ \\ Professor, Department of Computer Science and Engineering, MLRIT, Dundigal, Telangana ${ }^{2}$ \\ Assistant Professor, Department of Computer Science/Computer Applications, \\ Tiruvalluvar University Constituent College of Arts and Science, Attupakkam,Arakkonam- \\ 631051, India ${ }^{3}$
}

\begin{abstract}
Because of various appealing focal points, agreeable correspondences have been broadly viewed as one of the promising systems to enhance throughput and scope execution in remote interchanges. The hand-off hub (RN) assumes a key part in helpful interchanges, and $\mathrm{RN}$ determination may considerably influence the execution pick up in a system with agreeable media get to control (MAC). In this paper, we address the issue of RN choice while considering MAC overhead, which is brought about by handshake motioning as well as casing retransmissions because of transmission disappointment too. We outline a helpful MAC component with our ideal $\mathrm{RN}$ determination calculation, which is called ideal hand-off choice MAC, and utilize a hypothetical model. To investigate the collaboration execution picks up. We direct recreation tests in view of Network Simulator To assess our proposed agreeable MAC. Numerical outcomes approve the adequacy of our investigative model and demonstrate that our composed MAC fundamentally outflanks existing agreeable MAC components that don't consider retransmission MAC overhead.
\end{abstract}

\subsection{INTRODUCTION}

Agreeable correspondence is one of the quickest developing ranges of research, and it is probably going to be a key empowering innovation for effective range use in future. The key thought in client participation is that of asset sharing among numerous hubs in a system. The purpose for the investigation of client collaboration is that eagerness to impart power and calculation to neighboring hubs can prompt to reserve funds of general system assets. In conventional correspondence arranges, the physical layer is in charge of conveying data starting with one hub then onto the next. Conversely, client collaboration infers an outlook change, where the channel is not only one connection but rather the system itself.

In agreeable correspondences, autonomous ways between the client and the base station are created by means of the presentation of a transfer channel. The transfer channel can be considered as a helper channel to the immediate channel between the source and goal. A key part of the helpful correspondence process is the handling of the flag got from the source hub done 
by the transfer. These distinctive handling plans result in various helpful interchanges convention. Agreeable correspondences conventions can be for the most part sorted into settled transferring plans and versatile handing-off plans. In settled handingoff, the channelresources are partitioned between the source and the transfer in a settled (deterministic) way. The preparing at the transfer varies as indicated by the utilized convention

\section{CO-OPERATIVE MAC}

A MAC convention known as Coop MAC that takes into account handingoff in IEEE 802.11 systems was proposed additionally portrayed. The convention expands the appropriated coordination work (DCF) to fuse a hand-off hub in information trade. The RTS/CTS convention is stretched out with an "Assistant prepared To Send" (HTS) message.

The source chooses whether to utilize a hand-off in light of reserved data that shows if the hand-off decreases the broadcast appointment expected to convey the bundle to the goal. After the source sends a RTS message, the handoff can answer with a HTS message.

In the event that this HTS message is heard by the goal, then the goal answers with a CTS message that stores time for a two-bounce transmission. On the off chance that the source gets both CTS and HTS messages, the information parcel is sent to the transfer that then advances to the goal. In the event that the source hears just the CTS message, then the source sends specifically to the goal.

At the point when the goal recipient has a design like that in and is equipped for differing qualities joining, the hand-off utilizations a helpful coding plan, for example, that in . Notwithstanding, Coop MAC is in reverse perfect with existing 802.11 radios in that a similar convention messages and total air timemetric can be utilized regardless of the possibility that the beneficiary is not equipped for differences joining..

\section{AMPLIFY AND FORWARD METHOD}

Increase and-forward as a helpful flagging plan . Intensify and-forward is reasonably the most straightforward of the agreeable flagging technique. In this strategy, every client gets a boisterous form of the flag transmitted by its accomplice; the client then opens up and retransmits this loud flag .

The goal will join the data sent by the client and accomplice and will settle on a ultimate conclusion on the transmitted image. In spite of the fact that the clamor of the accomplice is opened up , the goal still gets two freely blurred renditions of the flag and is along these lines ready to settle on better choices for the transmitted images.

A potential test in this plan is that examining, intensifying, and retransmitting simple qualities might be mechanically non-minor. By and by, enhance and-forward is a basic strategy that fits examination, and in this way has been exceptionally valuable in advancing the comprehension of helpful correspondence framework.

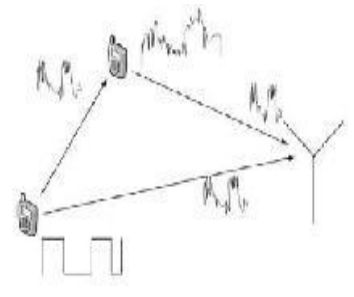

Fig. 1.1. Amplify and Forward method

\section{DECODE AND FORWARD}

METHOD:

Distinguish and-forward convention for client participation. These days a 
remote transmission is from time to time simple and the hand-off has enough registering power, so Detect and Forward is regularly the favored strategy to handle the information in the transfer. 


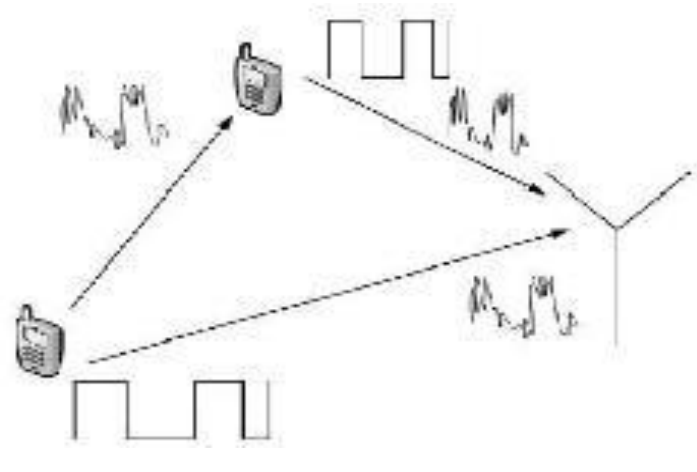

Fig. 1.2 .Decode and forward method

\subsection{PROPOSED SYSTEM}

The proposed ORS MAC, including outline structures, triangular handshaking to give collaboration, and the comparing participation table called Coop-table used to store the valuable participation data.

\section{COOPERATIVE MAC MECHANISM}

In traditional remote system MAC, for example, IEEE 802.11, when a hub S has information to transmit, it $\mathrm{s}$ if the transmitter battles and catches the channel successfully. The relating beneficiary D answers CTS to S after accepting the RTS. In the wake of accepting CTS, S starts sending information to $\mathrm{D}$, which will sustain ACK back to $S$ when the information are gotten effectively. Something else, a retransmission is performed to recoup the lost data.The helpful correspondence system can be connected in MAC with a few changes. In our proposed ORS MAC, a triangular handshake methodology is utilized to encourage helpful correspondence. We present three new sorts of edges, in particular,

Helpful Ready-To-Send (C-RTS)

Prepared To-Relay (RTR), and
Cooperative-Clear-To-Send (C-CTS)

In our helpful MAC Frame arrangements are appeared in Fig. 3.1 For similarity, all the edge arrangements depend on IEEE 802.11.

\section{RELAY SELECTION TECHNIQUES}

In our helpful MAC, a key issue is to figure out if to utilize agreeable correspondence and which hub is chosen as the RN. The response to both inquiries needs to analyze the execution pick up brought by helpful correspondence when an alternate $\mathrm{RN}$ is chosen. To this end, we have to infer the system throughput, considering MAC overhead and transmission disappointment.

The transmission disappointment is brought on by crash and channel transmission blunder. Since the transmission impact likelihood is not identified with participation, we expect that the transmission disappointment likelihood is equivalent to the transmission mistake likelihood in our proposed ideal $\mathrm{RN}$ choice calculation.

Let the transmission disappointment likelihood for direct transmission and agreeable transmission be signified by pd and pc, separately. Let the FTT for direct transmission and helpful transmission be signified by FTT_d and FTT_c, separately.

Considering transmission disappointment, the throughputs for immediate and helpful correspondence, which are meant we realize that participation could be received when 
T_c > T_d, on the grounds that the pick up of diminished transmission disappointment likelihood can cover the extra overhead of expanded FTT in collaboration mode. Not quite the same as existing agreeable MAC instruments, we select RN by considering FTT as well as transmission disappointment likelihood too. This is more reasonable in blunder inclined remote systems.

In ORS-MAC, when the execution pick up is higher than the overhead brought on by collaboration and the general throughput could be upgraded, agreeable transmission is received. The ideal $\mathrm{RN}$ is the one that can give greatest throughput in participation mode, and we can discover it as indicated by our proposed RN choice calculation. Considering retransmission brought on by transmission disappointment, the correct throughput could be computed by T_d and T_c , individually.

In our helpful MAC, RN is chosen by looking at the throughput for immediate and agreeable correspondence, i.e., $\mathrm{T}$ _d and T_c. The points of interest of the ideal RN choice In our helpful MAC, $\mathrm{RN}$ is chosen by contrasting the throughput for immediate and agreeable correspondence, i.e., T_d and T_c. The points of interest of the ideal $\mathrm{RN}$ determination calculation are demonstrated as follows, where RN_set means the arrangement of hubs, and T_set signifies the arrangement of throughput qualities gave by various RNs. The obliviousness of retransmission brought on by transmission disappointment in helpful MAC instruments may bring about the accompanying subsequences

As well as could be expected be missed

The agreeable open door could be squandered. The execution pick up of helpful correspondence is not completely misused

\section{Optimal Relay Selection Algorithm}

Optimal RN Selection Algorithm When $S$ needs to transmit data to $\mathrm{D}$

if $R N \_s e t \_=\varphi$ and $T \_d<T \_c$ then

direct transmission mode is adopted

end if

For calculating T_d and T_c, essential cooperation information should be provided. To this end, each node should maintain a table called Coop-table that keeps the useful cooperation information. There are six columns in the Coop-table: the ID of node, i.e., Gsd, Gsr, Grd, and Pr, and the count of sequential transmission failures of the corresponding node. CSI (Gsd,Gsr, and Grd) can be obtained by channel estimate.

According to the handshaking procedure and overhearing, each node can obtain Gsd, Gsr, Grd, and Pr and update the Coop-table. In fact, the Coop-table can be maintained in handshaking procedure without much additional overhead, which is similar. Therefore, the overhead of the Cooptable has been considered in the handshaking procedure.

At the point when any chose RN bombs in giving participation, the comparing disappointment check will be 
augmented by one. At the point when the disappointment tally achieves an edge that can be predefined, the data about this hub will be expelled from the Cooptable. The disappointment number will be reset to zero after an effective transmission.

To process the throughput, we should infer $\mathrm{pd}$ and $\mathrm{pc}$ first. As a rule, the blunder likelihood of the handshake technique can be disregarded on account of the short casing length and low essential transmission rate, and along these lines, the transmission mistake likelihood can be approximated to be the mistake likelihood of the information outline. For effortlessness, we expect that the remote channel is a Rayleigh blurring channel with Gaussian commotion, and bit mistakes are free indistinguishably appropriated over the entire edge. Without loss of sweeping statement, we expect that the regulation is paired stage move keying for effortlessness.

The transmission mistake likelihood of the information outline (with length information) at the goal in direct transmission mode is given by where information $b$ is indicated as the bit blunder rate (BER) of the information outline, which can be registered as information $\gamma \mathrm{sd}$ is the SNR of the gotten motion at $\mathrm{D}$ sent from $\mathrm{S}$, which can be figured as $\gamma \mathrm{sd}=\mathrm{PsGsd}_{\sigma} / \sigma 2$, and $\sigma 2$ is Gaussian clamor control. Thusly, as indicated by the length of information casing, the transmission blunder likelihood of that can be acquired.

\section{CO-OPERATION REGIONS}

In IEEE $802.11 \mathrm{~b}$, there are four distinctive transmission rates, i.e., 1, 2, 5.5, and $11 \mathrm{Mb} / \mathrm{s}$, and the comparing transmission reaches are produced in light of, and the connection between the got SNR and the transmission range is talked about as takes after .

Considering the Rayleigh blurring channel, the got SNR $\gamma$ at separation $\mathrm{d}$ has the accompanying dissemination: For instance, when the got SNR is lower than the base required SNR edge to bolster $5.5 \mathrm{Mb} / \mathrm{s}$ and higher than that of $2 \mathrm{Mb} / \mathrm{s}$, the transmission rate is 2 $\mathrm{Mb} / \mathrm{s}$. As indicated by the appropriation, we can get that the got SNR is influenced by separation and that the transmission rate is influenced by the gotten SNR. IEEE 802.11 backings diverse information transmission rates, which can be balanced by the transmission run. In $\mathrm{r} 1$, $\mathrm{r} 2, \mathrm{r} 5.5$, and r11 speak to the distinctive greatest transmission ranges, separately.

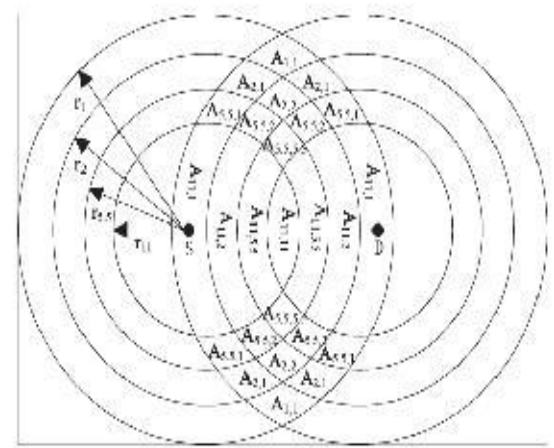

Fig. 3.4. Cooperation regions

Ax,y indicates the area where an $\mathrm{RN}$ can help the transmission using rate $\mathrm{x}$ and $\mathrm{y}$ megabits per second in two links, respectively, in cooperative communication. Assuming that the direct transmission rate between $\mathrm{S}$ and $\mathrm{D}$ is $2 \mathrm{Mb} / \mathrm{s}$ and $n$ nodes are uniformly 
distributed in the coverage area, then we can derive the probability of an RN located in Ax,y.

Srs,rd is the overlapping acreage of two circles, given that the distance between the center of the two circles is 1 , and their radii are $\mathrm{rs}$ and $\mathrm{rd}$, respectively,

\section{APPLICATIONS \\ Cooperative sensing for cognitive radio \\ Wireless Ad-hoc Network \\ Wireless Sensor Network \\ Vehicle-to-Vehicle \\ Communion}

\subsection{RESULTS AND DISCUSSION}

\section{SOFTWARE DESCRIPTION}

Network Simulator, A package of tools that simulates behavior of networks. Ns are a discrete event simulator targeted at networking research. Ns provides substantial support for simulation of $\mathrm{TCP}$, routing, and multicast protocols over wired and wireless (local and satellite) networks.

\section{SIMULATION RESULTS}

To verify our analytical model and validate the performance improvement of the proposed cooperative MAC mechanism, we use simulation experiments based on NS-2 to evaluate the throughput performance.

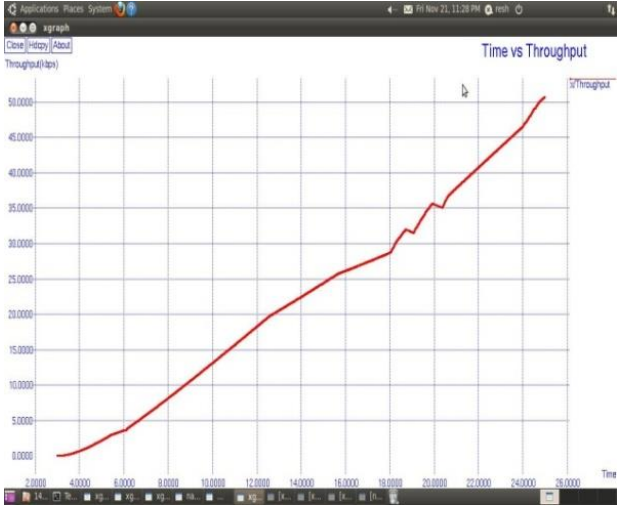

Fig. 4.1. Relationship Between Time versus Throughput

Fig.4.1 shows the performance throughput when the channel provided by the RN is better than the direct link. throughput of our proposed ORS-MAC it increases with the data frame length.

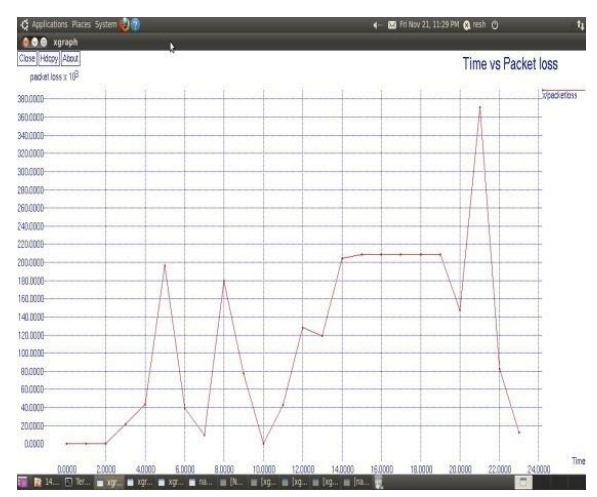

Fig. 4.2. Relationship between time versus packet loss

Fig.4.2 shows packet loss of generality, the better channel can provide a higher transmission rate. Packet loss can reduce throughput for a given sender, Losses between 5\% and $10 \%$ of the total packet stream will affect the quality significantly 


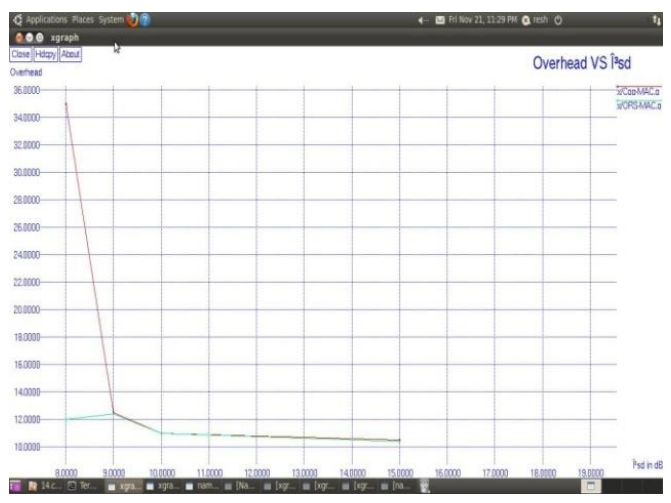

Fig. 4.3. Comparison between overhead versus SNR

fig.4.3 We compare the $E$ [overhead] and the signal to noise ratio (SNR) of ORS-MAC with that of Coop MAC. the overhead is caused by cooperation and thus we prefer direct transmission rather than the cooperation.

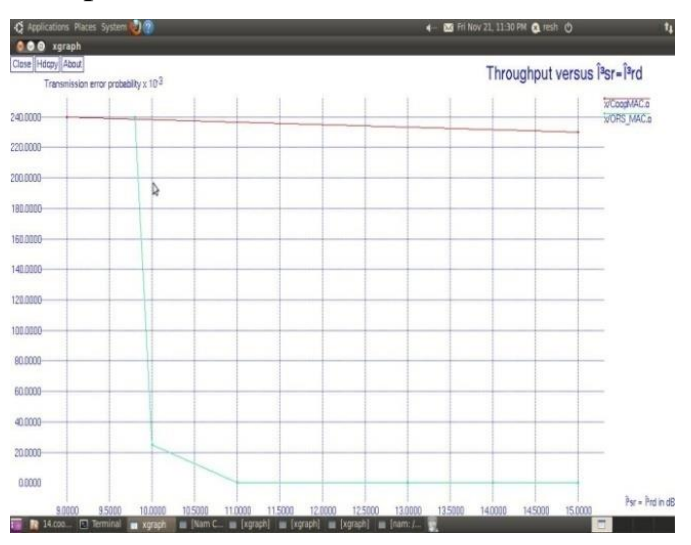

Fig. 4.4. Comparison between transmission error probability versus SNR

fig.4.4 shows the comparison of transmission error probability versus SNR at the destination between ORSMAC and Coop MAC. Although the transmission error probability can be declined. the transmission error probability of Coop MAC increases with the data frame length; in contrast, the transmission error probability of ORS-MAC keeps a lower value. the transmission error probability of cooperation is lower than that of direct transmission.

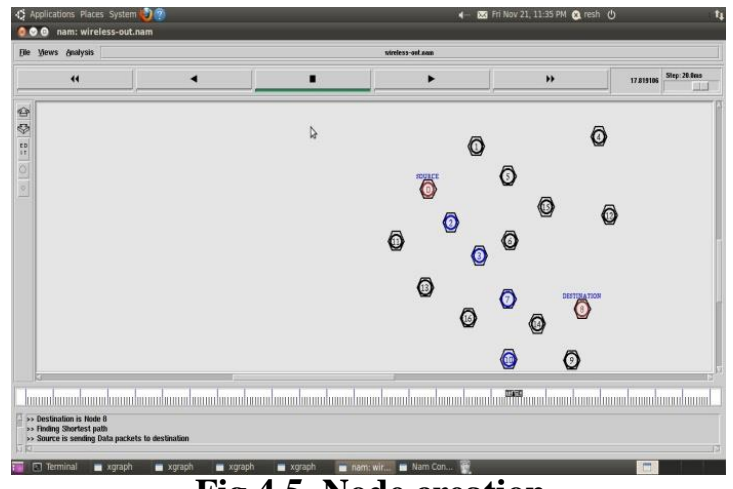

Fig.4.5. Node creation

fig.4.5 shows a node creation. The node can be created with its node type, size and properties. Created node can be located in the network. In this assume 16 nodes.

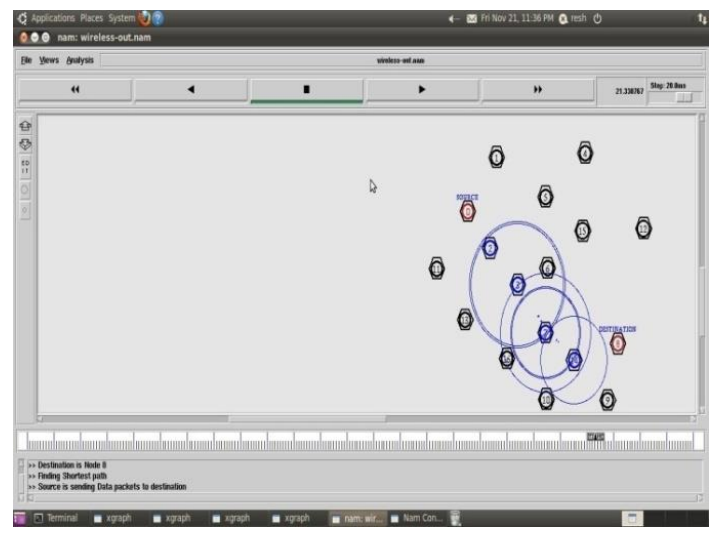

Fig. 4.7. Relay selection via packet transmission

fig.4.7 shows it is a moving environment we choose a best relay node the packet will be send to relay .The relay will send to the destination the above figure the packet will be send the node relay node 14 via destination. 


\subsection{CONCLUSION}

In this paper, we have proposed a hand-off choice calculation for an agreeable MAC component in a blunder inclined remote system to misuse the potential advantages by considering the MAC overhead brought about by retransmission. Through watchful convention plan, our proposed ORSMAC can choose the most reasonable hub as a hand-off, therefore easing the transmission disappointment likelihood and upgrading the participation opportunity.

Numerical outcomes have approved our logical model and demonstrated that ORS-MAC can altogether enhance arrange execution as far as throughput and reduction transmission blunder likelihood. Numerical outcomes show that ORSMAC beats traditional helpful MAC components without bringing about huge unpredictability overhead in framework outline..

The best possible determination of the transfer can successfully enhance the general execution of the system as far as higher information rate/through put. bring down power utilization and better piece blunder rate execution. agreeable remote systems, particularly on the execution of system layer.

\section{REFERENCES}

[1]Bin Cao, Yun Li, and Chonggang Wang, "Cooperative Media Access Control With

Optimal Relay Selection in Error-Prone Wireless Networks," IEEE Trans on vehicular technology, vol. 63, no. 1,january 2014.

[2]G. Bianchi, "Performance analysis of the IEEE 802.11 distributed coordination function," IEEE J. Sel. Areas Commun., vol. 18, no. 3, pp. 535-547, Mar. 2000

[3]B. Cao, G.Feng, and Y. Li, "Relay selection for cooperative MAC considering retransmission overhead," in Proc. IEEE GLOBECOM, Houston, TX, Dec. 2011, pp. 1-5.

[4]C.-T. Chou, J. Yang, and D.Wang, "Cooperative MAC protocol with automatic relay selection in distributed wireless networks," in Proc. Pervasive Comput. Commun. Workshops, New York, Mar. 2007, pp. 526-531.

[5]T. Guo and R. Carrasco, "CRBAR: Cooperative relay-based auto-rate MAC for multirate wireless networks," IEEE Trans. Wireless Commun., vol. 8, no. 12, pp. 5938-5947, Dec. 2009

[6] Rajesh, M. \& Gnanasekar, J.M. Wireless Pers Commun (2017) 97: 1267. https://doi.org/10.1007/s11277017-4565-9 
